\title{
An EASO Position Statement on Multidisciplinary Obesity Management in Adults
}

\author{
Volkan Yumuk $^{\mathrm{a}}$ Gema Frühbeck ${ }^{\mathrm{b}}$ Jean Michel Oppert ${ }^{\mathrm{c}}$ \\ Euan Woodward $^{d}$ Hermann Toplak $^{\mathrm{e}}$ for the Executive Committee of the \\ European Association for the Study of Obesity \\ a Department of Medicine, Division of Endocrinology, Metabolism and Diabetes, Istanbul \\ University Cerrahpasa Medical Faculty, Istanbul, Turkey, ${ }^{\mathrm{b}}$ Department of Endocrinology \\ and Nutrition, Clinica Univ. de Navarra, University of Navarra, CIBERobn, Instituto de Salud \\ Carlos III, Pamplona, Spain, 'Department of Nutrition, Pitie-Salpetriere Hospital, Institute \\ of Cardiometabolism and Nutrition (ICAN), University Pierre et Marie Curie, Paris, France, \\ ${ }^{\mathrm{d}}$ European Association for the Study of Obesity, London, UK, e Department of Medicine, \\ Medical University Graz, Graz, Austria
}

\section{Key Words}

Obesity management $\cdot$ OMTF $\cdot$ Weight loss $\cdot$ Multidisciplinary $\cdot$ Treatment $\cdot$ Statement

\begin{abstract}
Obesity has proven to be a gateway to ill health. It has already reached epidemic proportions becoming one of the leading causes of death and disability in Europe and world-wide. Obesity plays a central role in the development of a number of risk factors and chronic diseases like hypertension, dyslipidaemia and type 2 diabetes mellitus inducing cardiovascular morbidity and mortality. Therefore weight management plays a central role in controlling the respective risk factors and their consequences. Obesity is a complex condition of multifactorial origin. Biological but also psychological and social factors interfere to lead to excess body weight and its deleterious outcomes. Obesity management cannot focus any more only on weight (and BMI) reduction. More attention is to be paid to waist circumference (or waist-to-hip ratio, especially in females), the improvement in body composition (measured with body composition tracking systems like BOD POD, dual energy X-ray absorptiometry or bioelectrical impedance analysis) which is focusing on ameliorating or maintaining fat-free mass and decreasing fat mass. Management of co-morbidities, improving quality of life and well-being of obese patients are also included in treatment aims. This statement emphasises the importance of a comprehensive approach to obesity management.




\section{Introduction}

Obesity has proven to be a gateway to ill health. It has already reached epidemic proportions becoming one of the leading causes of death and disability in the world. Obesity plays a central role in the development of a number of risk factors and chronic diseases like hypertension, dyslipidaemia and type 2 diabetes mellitus inducing cardiovascular morbidity and mortality [1]. Therefore, weight management is going to play a major role in reducing morbidity and mortality of populations in Europe and world-wide. This statement emphasises the importance of a comprehensive approach to obesity management.

\section{Comprehensive Obesity Management}

Obesity is a complex condition of multifactorial origin. Biological but also psychological and social factors interfere to lead to excess body weight and its deleterious outcomes. Obesity management cannot focus any more only on weight (and BMI) reduction. More attention is to be paid to waist circumference (or waist-to-hip ratio, especially in females), the improvement in body composition (measured with body composition tracking systems like BOD POD, dual energy $\mathrm{X}$-ray absorptiometry or bioelectrical impedance analysis) which is focusing on ameliorating or maintaining fat-free mass and decreasing fat mass [2,3]. Management of co-morbidities, improving quality of life and well-being of obese patients are also included in treatment aims.

A comprehensive obesity management can only be accomplished by an appropriate obesity management team which is multidisciplinary and comprises different professionals able to tackle the different aspects of obesity and its related disorders. In accordance with this vision the European Association for the Study of Obesity (EASO) has developed a network of Collaborating Centres for Obesity Management (COMs) [2].

Appropriate goals of weight management emphasise realistic weight loss to achieve a reduction in health risks and should include promotion and maintenance of weight loss, and prevention of weight regain. Patients should understand that, since obesity is a chronic disease, weight management will need to be lifelong [3].

\section{Aims of Treatment}

\section{Management and Treatment of Obesity}

The management and treatment of obesity have wider objectives than weight loss alone and include risk reduction and health improvement. These may be achieved by modest weight loss, improved nutritional content of the diet and even modest increases in physical activity and fitness. Obesity management may reduce the need to treat co-morbidities with drugs. Appropriate treatment of obesity complications in addition to weight management should include: management of dyslipidaemia, optimising glycaemic control in patients with type 2 diabetes, normalising blood pressure in hypertension, management of respiratory disorders (such as sleep apnoea syndrome), attention to pain control and mobility needs in osteoarthritis, management of psychosocial disturbances, including affective disorders, eating disorders and low self-esteem, and body image disturbance. The socio-economic context of patients also needs to be taken into account in establishing the treatment strategy [3].

\section{Weight Objectives}

In some patients, especially in those who are overweight (BMI $25.0-29.9 \mathrm{~kg} / \mathrm{m}^{2}$ ), prevention of further weight gain (through dietary advice and increase in physical activity) 
Yumuk et al.: An EASO Position Statement on Multidisciplinary Obesity Management in Adults

rather than weight loss per se may be an appropriate target. Weight loss objectives should be realistic, individualised and aimed at the long term. A practical weight loss objective of $5-10 \%$ weight loss over a period of 6 months is realistic and has a proven health benefit. Improvements in waist circumference and/or body composition are alternative goals. A greater weight loss may be considered for those with greater degrees of obesity (BMI $\geq 35 \mathrm{~kg} / \mathrm{m}^{2}$ ). Maintenance of weight loss, prevention and treatment of co-morbidities are the three main criteria for success. Obesity is a chronic disease for which follow-up and continued supervision is necessary in order to monitor disease risks and treat co-morbidities (e.g., type 2 diabetes mellitus, cardiovascular disease) and prevent weight regain [3].

\section{The Present Status of Anti-Obesity Treatments}

Treatment should be individually tailored, and clinical care providers should be able to provide a wide spectrum of clinically proven treatment options and combinations of them, such as individual or group lifestyle modification (physical activity counselling, nutritional advice including structured intensive supervision, cognitive-behavioural therapy (CBT) and, in special cases, formula diets and other food replacement as well as supplements), antiobesity drug(s) and bariatric surgery.

\section{Induction of Lifestyle Intervention}

\section{Physical Activity}

Physical activity counselling is an integral part of obesity management. Physical activity may only have modest effects regarding weight reduction, but it brings important benefits regarding the preservation of fat-free mass during weight loss and promoting weight maintenance. Even small increases in physical activity can increase cardiorespiratory fitness and improve the cardiovascular risk factor profile. The impact on muscle mass of strength training will need adequate protein intake. The volume of physical activity estimated for weight maintenance appears higher than current recommendations for health maintenance in the general population. Physical activity counselling includes advice on both habitual physical activity in everyday life and structured supervised exercise. Exercise prescription must be tailored to the patients' ability and health status and focus on a gradual increase to levels that are safe [3].

\section{Dietary Prescription}

The use of a self-recorded food diary allows a qualitative assessment of the diet helping in advising individually tailored dietary modifications. In addition, it can be used to help the patient identify perceptions and beliefs about emotional eating behaviour (cognition) and eating habits (behaviour). Dietary advice should first encourage healthy eating as previously described. The management of eating disorders also appears as a pre-requisite before reduction in energy intake [3].

In the last years the use of formula diets has been increasingly popular for a subgroup of patients who feel in need of pre-prepared food with controlled quality. They may be used as very low calorie diet (VLCD) in full meal replacement plan or as a supplement to facilitate a low calorie diet (LCD). Similarly, there are full meals with an adequate macronutrient composition, which can be used in a treatment plan. VLCDs may have detrimental side effects and therefore should be instituted under a physician's supervision. VLCDs and LCDs are effective as long as they are used. Weight maintenance would need intermittent treatment with formula diet or drug therapy $[4,5]$. 
Yumuk et al:: An EASO Position Statement on Multidisciplinary Obesity Management in Adults

\section{Cognitive-Behavioural Approaches}

CBTs should be an integral part of every lifestyle modification. CBTs are techniques which aim to help a patient modify both his/her insight and understanding of thoughts and beliefs concerning weight regulation, obesity and its consequences; they also directly address behaviours that require change for successful weight loss and weight loss maintenance [6]. Common psychological problems like distress, frustration and depression are co-factors of overeating. As a consequence the feeling of guilt is leading to a vicious cycle where eating becomes a reward.

Randomised controlled trials have provided evidence that clinically significant weight loss and decisive benefits on cardiometabolic risk factors can be obtained via lifestyle changes in the course of an intensive, multicomponent programme for adults with obesity [7, 8]. Structured programmes of intensive behavioural therapy for adult obesity should include regular face-to-face meetings over a year, with most of the encounters in the first few months to check with the patient the best way to achieve realistic goals in an adequate time frame. In spite of the numerous scheduled meetings, these programmes have been shown to be a cost-effective approach from a health funder and societal perspective [9]. No direct evidence has been found that weight loss interventions are associated with an elevated risk for weight cycling, depression or eating disorders [10].

\section{Pharmacotherapy}

Pharmacological treatment should be considered as part of a comprehensive strategy of disease management [11]. Pharmacotherapy can help patients to maintain compliance, ameliorate obesity-related health risks and improve quality of life. It can also help to prevent the development of obesity co-morbidities (e.g. type 2 diabetes mellitus). Current drug therapy is recommended for patients with a BMI $\geq 30 \mathrm{~kg} / \mathrm{m}^{2}$ or a BMI $\geq 27 \mathrm{~kg} / \mathrm{m}^{2}$ with an obesity-related disease (e.g. hypertension, type 2 diabetes mellitus). Drugs should be used according to their licensed indications and restrictions. The efficacy of pharmacotherapy should be evaluated after the first 3 months of therapy (responders). If weight loss achieved is considered satisfactory ( $>5 \%$ weight loss in patients without and $>3 \%$ in patients with diabetes), treatment should be continued. Improvements in waist circumference and/or body composition could be used as alternative, more realistic, indicators for success. Treatment should be discontinued in non-responders [3].

Currently, there are few options for obesity pharmacotherapy. The only available approved drug for weight management in Europe is orlistat. This triglyceride lipase inhibitor reduces fat absorption by $30 \%$. In combination with a hypocaloric low-fat diet it is adding a modest effect to the lifestyle intervention. Orlistat is available in two dosages: $120 \mathrm{mg}$ tid (prescription) and $60 \mathrm{mg}$ tid (OTC). The published data demonstrate efficacy and safety over a period of 4 years $[12,13]$. A number of other drugs is currently tested in clinical trials for weight management worldwide [14]. Such medications could fill the gap between current treatment options and patients' needs.

\section{Bariatric Surgery}

Bariatric surgery is the most effective measure for inducing substantial weight loss in carefully selected patients. Surgery should be seen as part of a lifelong weight management programme and follow-up of medical complications. Current evidence points to major benefits in terms of prevention of type 2 diabetes, important gains regarding cardiovascular risk and cancer reduction (in women), and suggests increased longevity [15]. The respective guide- 
Yumuk et al.: An EASO Position Statement on Multidisciplinary Obesity Management in Adults

lines for metabolic and bariatric surgery have been previously published [16, 17]. For a given patient, the risk-benefit ratio needs to be carefully assessed by an experienced multidisciplinary team including the surgeon.

Today, the most common surgical techniques are restrictive procedures (that limit food ingestion) such as adjustable gastric banding (AGB), and sleeve gastrectomy (SG). Operations limiting absorption of macronutrients (limiting energy absorption) are gastric bypass (GBP), biliopancreatic diversion (BPD) or combined operations such as biliopancreatic diversion with duodenal switch (BPD-DS). The expected average weight loss and long-term weight maintenance is increasing with the following procedures: AGB, SG, GBP, BPD-DS, BPD. However, the surgical complexity and potential surgical and long-term nutritional risks of the procedures increase in the same order [3]. The preparation before surgery and the follow-up after surgery are part of the comprehensive approach described here and further detailed in the European Interdisciplinary Guidelines $[16,17]$.

\section{Social Issues}

In addition to the social gradient in obesity prevalence, social inequalities represent strong barriers for the delivery of high-quality health care to obese patients. Obese individuals are subject to a high level of stigmatization resulting in discrimination. Reducing weight stigma and promoting access to healthier lifestyle options without blame are crucial in weight management. It may be difficult to assess the responsibility of an individual for obesity or future weight gain, but the current trends are suggesting a responsibility which is shared by both the society and the obese individual (co-responsibility) [18].

Financially and physically accessible primary care for delivery of preventive interventions and for early detection and treatment of non-communicable diseases is mandatory. Dietary risk factors and physical inactivity form a heavy burden on society by increasing premature deaths and leading to higher disability rates [19]. Society has to develop lifelong strategies to promote healthy lifestyles which might include teaching of nutritional knowledge and physical activity starting at an early age. Adjusting prices for healthy food and hydration; promoting food labelling, restricting advertisement on unhealthy food and beverages, banning unhealthy food in schools and providing easy access to free facilities for physical activity in a safe environment must become priorities $[1,18]$.

\section{Conclusion}

While acknowledging the importance of prevention in tackling the obesity epidemic, the adult obese patients suffering from this disease and the application of currently available effective approaches merit equal attention. Since obesity is a gateway to many other diseases its appropriate prevention and management will block a major supply to ill health [1]. Obesity management is multidisciplinary by nature and requires fine co-operation between professionals with complementary expertise. Delivering comprehensive treatment to those who are obese represents a major challenge for health care systems. EASO therefore asks member states and COMs to help advance the co-ordination of obesity management and research in Europe to the benefits of citizens, society and industry.

\section{Disclosure Statement}

The authors have no disclosures to declare. 
Yumuk et al.: An EASO Position Statement on Multidisciplinary Obesity Management in Adults

\section{References}

1 Frühbeck G, Toplak H, Woodward E, Yumuk V, Maislos M, Oppert JM: Obesity: the gateway to ill health - an EASO position statement on a rising public health, clinical and scientific challenge in Europe. Obes Facts 2013; 6:117-120.

-2 Tsigos C, Hainer V, Basdevant A, Finer N, Mathus-Vliegen E, Micic D, Maislos M, Roman G, Schutz Y, Toplak H, Yumuk V, Zahorska-Markiewicz B: Criteria for EASO-collaborating centres for obesity management. Obes Facts 2011;4:329-333.

3 Tsigos C, Hainer V, Basdevant A, Finer N, Fried M, Mathus-Vliegen E, Micic D, Maislos M, Roman G, Schutz Y, Toplak H, Zahorska-Markiewicz B: Management of obesity in Adults: European Clinical Practice Guidelines. Obes Facts 2008;1:106-116.

4 Saris WH: Very low calorie diets and sustained weight loss. Obes Res 2001;9:295S-310S.

5 Heymsfield SB, van der Mierlo CA, van der Knaap HC, Heo M, Frier HI: Weight management using a meal replacement strategy: meta and pooling analysis from six studies. Int J Obes Relat Metab Disord 2003;27: 537-549.

6 Sharma M: Behavioral interventions for preventing and treating obesity in adults. Obes Rev 2006;7:183-200.

7 Jebb SA, Ahern AL, Olson AD, Aston LM, Holzapfel C, Stoll J, Amann-Gassner U, Simpson AE, Fuller NR, Pearson S, Lau NS, Mander AP, Hauner H, Caterson ID: Primary care referral to a commercial provider for weight loss treatment versus standard care: a randomised controlled trial. Lancet 2011;137:1485-1492.

8 Jolly K, Lewis A, Beach J, Denley J, Adab P, Deeks JJ, Daley A, Aveyard P: Comparison of range of commercial or primary care led weight reduction programmes with minimal intervention control for weight loss in obesity: lighten up randomised controlled trial. Br Med J: 2011;343:d500.

-9 Fuller NR, Colagiuri S, Schofield D, Olson AD, Shrestha R, Holzapfel C, Wolfenstetter SB, Holle R, Ahern AL, Hauner H, Jebb SA, Caterson ID: A within-trial cost-effectiveness analysis of primary care referral to a commercial provider for weight loss treatment, relative to standard care - an international randomised controlled trial. Int J Obes (Lond) 2013;37:828-834.

10 Frühbeck G. Obesity: Screening for the evident in obesity. Nat Rev Endocrinol 2012;8:570-572.

11 Hainer V, Toplak H, Mitrakou A: Treatment modalities of obesity: what fits whom? Diabetes Care 2008; 31(suppl 2): S269-S277.

12 Torgerson JS, Hauptman J, Boldrin MN, Sjöström L: XENical in the prevention of diabetes in obese subjects (XENDOS) study: a randomized study of orlistat as an adjunct to lifestyle changes for the prevention of type 2 diabetes in obese patients. Diabetes Care 2004;27:155-161.

13 Toplak H, Ziegler O, Keller U, Hamann A, Godin C, Wittert G, Zanella MT, Zúñiga-Guajardo S, Van Gaal L: X-PERT: weight reduction with orlistat in obese subjects receiving a mildly or moderately reduced energy diet: early response to treatment predicts weight maintenance. Diabetes Obes Metab. 2005; 7:699-708.

14 Witkamp RF: Current and future drug targets in weight management. Pharm Res 2011;28:1792-1818.

15 Sjöström L: Review of the key results from the Swedish Obese Subjects (SOS) trial - a prospective controlled intervention study of bariatric surgery. J Intern Med 2013;273:219-234.

16 Fried M, Yumuk V, Oppert JM, Scopinari N, Torres AJ, Weiner R, Yashkov Y, Frühbeck G: IFSO-EC and EASO Interdisciplinary European guidelines on metabolic and bariatric surgery. Obes Facts 2013;6:449-468.

17 Fried M, Yumuk V, Oppert JM, Scopinari N, Torres AJ, Weiner R, Yashkov Y, Frühbeck G: IFSO-EC and EASO Interdisciplinary European Guidelines on metabolic and bariatric surgery. Obes Surg 2014;24:42-55.

18 Sikorski C, Luppa M, Kaiser M, Glaesmer H, Schomerus G, König HH, Riedel-Heller SG: The stigma of obesity in the general public and its implications for public health - a systematic review. BMC Public Health 2011;11: 661.

19 Di Cesare M, Khang Y-H, Asaria P, Blakely T, Cowan MJ, Farzadfar F, Guerrero R, Ikeda N, Kyobutungi C, Msyamboza KP, Oum S, Lynch JW, Marmot MG, Ezzati M, on behalf of The Lancet NCD Action Group: Inequalities in non-communicable diseases and effective responses. Lancet 2013;381:585-597. 